\title{
SIMULAÇÕES COM PYTHON E VIDEOANÁLISE DO MOVIMENTO BALÍSTICO COM RESISTÊNCIA DO AR
}

DOI: 10.37702/2175-957X.COBENGE.2021.3408

Rafael Zacarias Palierini - rafael.palierini.contato@gmail.com

Centro Universitário FEI

Rua da Abolição 64

09632-080 - São Bernardo do Campo - SP

Eliane F Chinaglia - echinaglia@fei.edu.br

Centro Universitário FEI

Av Humberto de alencar Castelo Branco 3972

09850-901 - São Bernardo do Campo - SP

Sueli Hatsumi Masunaga - sueli.masunaga@gmail.com

Centro Universitário FEl

R. Cristiano Angeli 1737

09812-601 - São Bernardo do Campo - SP

Resumo: $O$ objetivo deste trabalho é apresentar uma atividade para alunos ingressantes que esteja alinhada com o desenvolvimento de competências na formação em engenharia. Propomos o estudo do movimento balístico, em que a resistência do ar pode ser considerada na equação de movimento, através de um programa de simulação desenvolvido em Python em conjunto com videoanálise. Como ilustração, apresentamos o estudo do movimento de cinco bolas diferentes lançadas obliquamente. A visualização conjunta de dados experimentais e simulados no programa Python permitiu analisar a relevância ou não do efeito da resistência do ar no movimento de cada uma das bolas. A resistência do ar foi desprezível no estudo do movimento das bolas de basquete e futebol. Entretanto, foi preciso considerar uma força viscosa atuando no sistema para a descrição dos movimentos das bolas de isopor, plástico e vôlei. Nesses casos, também estimamos o coeficiente de arrasto quantitativamente através da simulação.

Palavras-chave: Simulação em Python. Movimento balístico. Força de arrasto. 


\section{SIMULAÇÕES COM PYTHON E VIDEOANÁLISE DO MOVIMENTO BALÍSTICO COM RESISTÊNCIA DO AR}

\section{INTRODUÇÃO}

Nos últimos anos, uma nova perspectiva no ensino em engenharia vem sendo discutida e trabalhada no sentido de assegurar o desenvolvimento por competências estabelecidas nas Diretrizes Curriculares Nacionais do Curso de Graduação em Engenharia no perfil do egresso (CNE, 2019). Nesse contexto, o desenvolvimento gradual e crescente, ao longo do curso, de habilidades relacionadas à pesquisa, desenvolvimento, adaptação e utilização de novas tecnologias; à capacidade de reconhecer as necessidades dos usuários; à implantação de solução de problemas complexos e mal estruturados; à capacidade de atuação e adaptação às novas demandas da sociedade e do mundo do trabalho, dentre outras, tem um papel fundamental na formação do engenheiro atual, com perfil criativo, cooperativo, crítico, ético, etc. (CNE, 2019). Nesta visão, um processo de ensino-aprendizagem ativo centrado no aluno é fundamental para contribuir para esse desenvolvimento, além de promover o protagonismo, o envolvimento e a responsabilidade do estudante no seu próprio aprendizado (ROGERS,1983).

Em particular, a competência geral em analisar e compreender os fenômenos físicos e químicos pode ser desenvolvida desde o início do curso de engenharia, pelos alunos ingressantes, com a aplicação de atividades experimentais envolvendo simulação e tecnologia da informação e comunicação (TIC) no curso de Física I. O desenvolvimento de simulações que complementem a análise experimental e estejam diretamente interligadas ao conteúdo teórico, leva o aluno a assumir um papel mais ativo, pois, além da realização do experimento, ele é induzido a buscar o embasamento teórico para a compreensão dos resultados da simulação e correta comparação deste com os dados experimentais, tornando a aprendizagem mais dinâmica. Além disso, as simulações podem estimular a utilização de linguagens de programação em disciplinas de física, facilitar o aprendizado do aluno em assuntos conceitualmente mais desafiadores e ajudar na solução de equações com cálculos analíticos mais complexos (DORNELES; ARAUJO; VEIT, 2006).

Assim, o objetivo deste artigo é propor uma metodologia para a aquisição e análise de dados envolvendo o movimento balístico, em que é possível determinar a relevância ou não da resistência do ar na descrição do movimento de um determinado objeto. Por exemplo, a partir da aquisição de dados através da videoanálise de um movimento balístico, os alunos podem comparar os dados experimentais com a simulação, obtendo assim os valores dos parâmetros associados à força viscosa. Em função das análises realizadas, os alunos podem avaliar os limites de aplicabilidade de modelos teóricos e da própria simulação, possibilitando a reflexão crítica do procedimento e análise experimental ao longo da experiência. Para ilustrar a metodologia aqui proposta, mostramos ao longo deste artigo as funcionalidades do programa que desenvolvemos e a análise do movimento balístico de cinco bolas com massas e diâmetros diferentes com o objetivo de avaliar os parâmetros associados à força viscosa.

Assim, o objetivo deste artigo é propor o estudo do movimento balístico, através da utilização de dados experimentais com videoanálise e simulação em Python. Com isso, é possível abordar desde o caso mais simples, de um lançamento balístico ou queda livre sem resistência do ar, até um caso mais complexo e realista, em que a resistência do ar é considerada no movimento, envolvendo a solução numérica de equações diferenciais não lineares. Isso se torna possível através da modelagem computacional, lembrando que o 
efeito da resistência do ar, em geral, é pouco ou nunca abordado nos cursos iniciais de física no ensino superior, devido à complexidade matemática envolvida. O tema escolhido também é relevante na área de engenharia, pois o entendimento do movimento dos projéteis sob o efeito da resistência do ar está relacionado com a modelagem de veículos de passeio, esportivos e de carga, com lançamento de foguetes, com balística e com desobstrução de passagens utilizando explosivos (FREIRE et al., 2016).

\section{EQUAÇÕES DE MOVIMENTO}

\subsection{Movimento balístico sem resistência do ar}

O movimento balístico sem resistência do ar é abordado no ensino médio, assim como nos primeiros semestres dos cursos de engenharia. Neste caso, somente a força Peso atua na partícula e as equações de movimento, $x(t)$ e $y(t)$, são dadas pelas Equações (1) e (2) e a equação da trajetória pela Equação (3). Nessas expressões, o eixo $y$ está orientado para cima e $\theta_{o}$ é o ângulo medido em relação ao eixo $x$ positivo, onde $g$ é a aceleração gravitacional e $v_{o}$ é a velocidade inicial de lançamento (HALLIDAY; RESNICK; WALKER, 2016).

$$
\begin{gathered}
x-x_{0}=v_{0 x} t\left(\operatorname{com} v_{0 x}=v_{0} \cos \theta_{0}\right) \\
y-y_{0}=v_{0 y} t-\frac{1}{2} g t^{2}\left(\operatorname{com} v_{0 y}=v_{0} \operatorname{sen} \theta_{0}\right) \\
y(x)=\left(\tan \theta_{0}\right) x-\frac{g x^{2}}{2\left(v_{0} \cos \theta_{0}\right)^{2}}
\end{gathered}
$$

\subsection{Movimento balístico com resistência do ar}

Uma das bases do programa desenvolvido neste trabalho considera uma força de atrito linearmente proporcional à velocidade do objeto. Neste caso, além da força Peso, uma força viscosa,

$$
\overrightarrow{F_{v}}=-b \cdot \vec{v}
$$

atua no objeto, onde $b$ é a constante de amortecimento. Comparando com a Lei de Stokes, $\vec{F}=-6 \pi \eta r \vec{v}$, a constante $b$ é escrita como

$$
b=6 \pi \eta r
$$

onde $\eta$ é a viscosidade do meio e $r$ o raio associado à área da seção transversal da partícula.

Neste caso, a dedução das equações de movimento pode ser encontrada no artigo de Freire et al. (2016) e são dadas pelas Equações (6) - (8), onde $\beta=b / m$. A existência de uma solução analítica permite o cálculo da simulação de uma forma bastante simples.

$$
x(t)=v_{0} \cos \theta_{0}\left(\frac{1-e^{-\beta t}}{\beta}\right)
$$




$$
\begin{gathered}
y(t)=\left(v_{0} \operatorname{sen} \theta_{0}+\frac{g}{\beta}\right)\left(\frac{1-e^{-\beta t}}{\beta}\right)-\frac{g}{\beta} t \\
y(x)=\left(\tan \theta_{0}+\frac{g}{\beta v_{0} \cos \theta_{0}}\right) x+\frac{g}{\beta^{2}} \ln \left(\frac{1-\beta x}{v_{0} \cos \theta_{0}}\right)
\end{gathered}
$$

Uma segunda base no desenvolvimento desse programa considera, além da força viscosa dada pela Equação (4), uma força viscosa proporcional à velocidade ao quadrado expressa por

$$
\vec{F}_{v^{2}}=-c \cdot|v| \vec{v}
$$

Aqui, $c$ pode ser obtido comparando essa equação com a força de arrasto, $F_{a}=\frac{1}{2} C \rho A v^{2}$, de modo que

$$
c=\frac{1}{2} C \rho A,
$$

onde $C$ é o coeficiente de arrasto, $\rho$ é a densidade volumétrica do meio e $A$ a área da seção transversal.

O estudo do movimento considerando a resistência do ar linearmente proporcional à velocidade do objeto entrega resultados mais condizentes com a realidade somente no caso de velocidades muito baixas. De modo mais geral, para velocidades da ordem de $\mathrm{m} / \mathrm{s}$ a inferiores à velocidade do som, a resistência do ar é melhor representada pela dependência da velocidade ao quadrado da força viscosa (FERREIRA, 2001; LOCK, 1982). Um valor de referência para a dependência da força viscosa com a velocidade foi determinado por James A. Lock (1982) como sendo similar à $v r=1,5 \mathrm{~m}^{2} / \mathrm{s}$. Em outras palavras, a Equação (4) é válida quando $v r<1,5 \mathrm{~m}^{2} / \mathrm{s}$ e a Equação (9) é válida quando vr $>1,5 \mathrm{~m}^{2} / \mathrm{s}$.

Levando em consideração as forças viscosas apresentadas nas Equações (4) e (9), a segunda Lei de Newton é dada pela equação:

$$
\vec{a}=\frac{d \vec{v}}{d t}=-g \hat{\jmath}-\beta\left(v_{x} \hat{\imath}+v_{y} \hat{\jmath}\right)-\gamma\left(v_{x}^{2}+v_{y}^{2}\right)^{\frac{1}{2}}\left(v_{x} \hat{\imath}+v_{y} \hat{\jmath}\right),
$$

onde, $\beta=b / m$ e $\gamma=c / m$ ou

$$
\gamma=\frac{1}{2} \frac{C \rho A}{m}
$$

\section{PROGRAMA DE SIMULAÇÃO}

Neste trabalho, a simulação do movimento balístico foi desenvolvida em Python, com a utilização da documentação oficial da linguagem no Python Docs (PYTHON, 2021). Além dessa linguagem de programação já estar presente na grade curricular de Engenharia e ser open-source, o fator principal que contribuiu para a sua escolha foi o fato do Python ser uma linguagem simples e ao mesmo tempo muito poderosa, sendo excelente para quem 
está começando a programar. Por essa razão, seu uso vem crescendo consideravelmente ao longo dos anos (SRINATH, 2017).

As bibliotecas utilizadas na simulação foram: matplotlib, numpy, os, sys, time, tkinter e webbrowser. Todas essas bibliotecas já vêm pré-instaladas na plataforma Anaconda Navigator (ANACONDA, 2021), o que facilita a execução do programa de simulação tanto para os usuários iniciantes quanto para os mais experientes. Vale destacar que o tkinter é uma ótima biblioteca de interface gráfica para iniciantes em programação e com documentação no Python Docs (PYTHON, 2021). Essa biblioteca permite que os gráficos sejam atualizados conforme a interação do usuário com a interface, tornando o programa mais amigável e intuitivo. Com o objetivo de contribuir para o estudo do movimento balístico, a simulação desenvolvida neste trabalho está gratuitamente disponível para download, em um repositório GitHub (PALIERINI, 2021)

\section{METODOLOGIA}

\subsection{Aquisição de dados experimentais por videoanálise}

Para análise do movimento balístico, os dados da posição como função do tempo foram obtidos para a trajetória de cinco bolas, de diferentes diâmetros e massas, indicados na Tabela 1. Todas as bolas foram lançadas manualmente por uma pessoa em dois locais distintos, o primeiro em campo aberto para as bolas de basquete, futebol e vôlei e o segundo em um ginásio para as bolas de isopor e de plástico. Inicialmente, a trajetória de cada bola foi gravada na resolução de $1280 \times 720$ pixels (720p HD) à taxa de 30 quadros por segundo (30 fps). Em seguida, os vídeos foram analisados no programa livre Tracker Video Analysis and Modeling Tool (PHYSICS, 2021), para a obtenção da posição da bola como função do tempo. Com a realização da videoanálise pelo programa Tracker, é possível obter, dentre outros parâmetros, os dados da posição $[x(t), y(t)]$, velocidade $\left[v_{x}(t), v_{y}(t)\right]$ e aceleração $\left[a_{x}(t), a_{y}(t)\right]$ do objeto ao longo dos eixos $x$ e $y$, quadro a quadro. Finalmente, os dados $x(t), y(t), v_{x}(t), v_{y}(t), a_{x}(t)$ e $a_{y}(t)$ foram exportados no formato .csv (commaseparated values) para serem importados no programa de simulação.

A partir da análise dos dados da trajetória, foram obtidos a velocidade inicial $\left(v_{0}\right)$ e 0 ângulo de lançamento $(\theta)$ de cada bola no próprio Tracker, mostrados na Tabela 1, para utilizá-los como parâmetros iniciais na simulação. Em alguns casos, os dados de $v(t)$ e $a(t)$ podem flutuar consideravelmente: pequenas flutuações na determinação da posição, seja manualmente ou automaticamente (auto-tracker), podem acarretar maiores flutuações na velocidade e maiores ainda na aceleração.

Tabela 1 - Dados experimentais das bolas utilizadas neste trabalho.

\begin{tabular}{c|c|c|c|cc}
\hline Tipo da bola & Raio & Massa & Velocidade inicial & \multicolumn{2}{|c}{ Ângulo de lançamento } \\
& $\left(10^{-2} \mathrm{~m}\right)$ & $\left(10^{-3} \mathrm{~kg}\right)$ & $(\mathrm{m} / \mathrm{s})$ & 3, & 34 \\
\hline Bolinha de isopor & 1,7 & 0,603 & 3,3 & 122 & 2,13 \\
\hline Bolinha de plástico & 2,9 & 22,244 & 8,2 & 122 & 2,13 \\
\hline Bola de futebol & 15,4 & 420,0 & 6,6 & 121 & 2,11 \\
\hline Bola de vôlei & 13,0 & 268,0 & 8,5 & 56 & 0,98 \\
\hline Bola de basquete & 15,2 & 521,0 & 6,2 &
\end{tabular}

Fonte: Autores

É importante comentar que, atualmente, a maioria dos alunos possuem smartphones, que suportam gravações à $30 \mathrm{fps}$ e superiores, assegurando a realização da parte experimental. A atividade ainda pode ser estendida e associada à cultura maker (HALVERSON; SHERIDAN, 2014), em que os alunos poderão construir protótipos de 
foguete, dispositivos de lançamento, como a catapulta caseira, e outros projetos para o estudo do movimento. Como podem ser facilmente realizados, com custo praticamente nulo e, inclusive, em casa pelos estudantes, a atividade proposta pode proporcionar 0 desenvolvimento das competências citadas na Introdução mesmo com a virtualização das aulas em meio à pandemia da COVID-19 ou na modalidade de educação à distância (EAD).

\subsection{Desenvolvimento e aplicação da simulação}

A simulação foi desenvolvida para que o movimento balístico possa ser analisado considerando-se dois métodos: (i) Método analítico: a força viscosa é proporcional somente à $v$. Neste caso a obtenção das grandezas cinemáticas é feita através das Equações (6) (8). (ii) Método numérico: a força viscosa é proporcional à $v$ e $v^{2}$ do objeto. A Equação (11) é uma equação diferencial não linear e, portanto, não possui solução analítica. Em vista disso, recorremos à resolução numérica, utilizando o método numérico de Runge-Kutta de 4a ordem para a obtenção das grandezas cinemáticas (HOFFMAN; FRANKEL, 2018). Nos dois casos, é possível gerar uma curva simulada do movimento sem resistência do ar inserindo "0" (zero) nos parâmetros relacionados à força viscosa ( $\beta$ e $\gamma)$. Para que a utilização da simulação seja facilmente visualizada, apresentamos a seguir algumas funcionalidades do programa.

A Figura 1 apresenta as telas iniciais do programa de simulação para a análise pelo (a) "Método Numérico", que pode ser acessado pelo botão "Fv $\propto \mathbf{v}$, v2" e (b) "Método Analítico", que pode ser acessado pelo botão "Fv $\propto$ v". Os símbolos e as unidades dos parâmetros presentes na simulação estão especificados no Quadro 1, sendo que alguns deles não aparecem na tela inicial da simulação. A imagem no canto inferior direito tem o propósito de mostrar o sistema de referência que deve ser considerado, especialmente para a inserção do valor de $\theta$. Na opção "AJUDA", é possível encontrar informações sobre a simulação e, também, um botão com o ícone do GitHub, que ao ser clicado nos redireciona automaticamente para a página do repositório oficial na plataforma.

Os dados experimentais obtidos pelo Tracker (arquivo tipo .csv) são inseridos na simulação clicando primeiro em "TRAJETÓRIA" e depois em "IMPORTAR". Em seguida, os gráficos são plotados automaticamente pela simulação. Ao lado esquerdo da Figura $1 a-b$ é possível ver uma lista de botões, que vai de "TRAJETÓRIA" até "AJUDA". Cada botão é responsável por selecionar e alternar para a grandeza que se deseja observar no gráfico. A simulação é mostrada juntamente com os dados experimentais para que as curvas geradas sejam imediatamente e facilmente comparadas. As curvas são simuladas através da inserção de todos os parâmetros na parte central, desde "NOME" até "LIMITE DE QUEDA". Para iniciar, uma legenda deve ser digitada no campo "NOME". Para simular uma curva sem atrito, o valor " 0 " deve ser digitado nos campos " $\beta$ " e " $\gamma$ ". Os valores em " $\theta$ " e " $v_{0}$ " são aqueles obtidos pelo Tracker, que constam na Tabela 1 , mas que podem ser levemente modificados, dentro da precisão de medida do Tracker, para que a curva simulada se adeque à curva experimental no início do movimento. Os parâmetros restantes podem ser inseridos de acordo com as especificações do Quadro 1. Após a inserção de todos os valores, a curva simulada é gerada clicando em "CALCULAR".

Figura 1 - Interfaces da simulação pelo (a) "Método Numérico" e (b) "Método Analítico". 

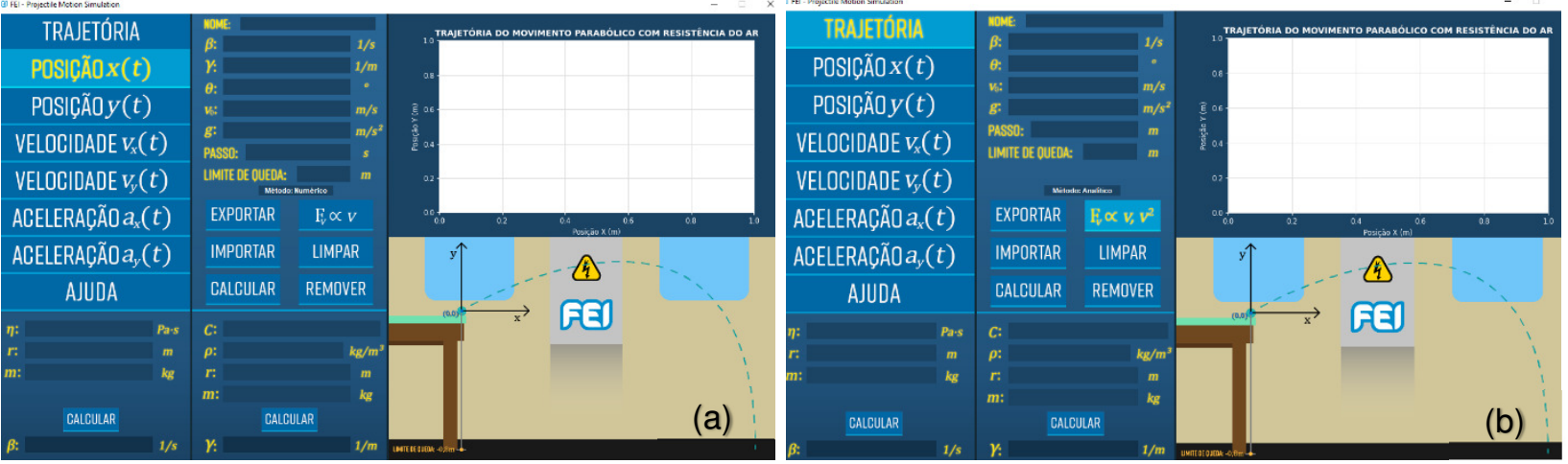

Fonte: Autores

Quadro 1 - Parâmetros das equações do movimento na simulação em Python.

\begin{tabular}{|c|c|c|}
\hline Símbolo & Tipo de parâmetro & Unidade \\
\hline$\beta$ & Parâmetro de atrito do ar $\propto v$ & $1 / \mathrm{s}$ \\
\hline$\gamma$ & Parâmetro de atrito do ar $\propto v^{2}$ & graus \\
\hline$\theta$ & Ângulo de lançamento & $\mathrm{m} / \mathrm{s}$ \\
\hline$v_{0}$ & Velocidade inicial & $\mathrm{m} / \mathrm{s}^{2}$ \\
\hline$g$ & Aceleração da gravidade & $\mathrm{s}$ \\
\hline$t_{0}$ & Tempo inicial & $\mathrm{s}$ \\
\hline$t_{1}$ & Tempo final & $\mathrm{m}$ \\
\hline Passo (Método analítico) & Espaço entre pontos para simulação & $\mathrm{s}$ \\
\hline Passo (outras trajetórias) & Espaço entre pontos para simulação & $\mathrm{m}$ \\
\hline Limite de queda & Menor valor da posição y após a esfera ser lançada & $\mathrm{s}$ \\
\hline Limite de tempo & Tempo máximo que a simulação irá percorrer a partir & $\mathrm{m}$ \\
\hline$r$ & do instante inicial com $t_{0}=0$ & $\mathrm{mg}$ \\
\hline$m$ & Raio da esfera & Pa.s \\
\hline$\eta$ & Massa da esfera & "adimensional” \\
\hline$C$ & Viscosidade do meio & $\mathrm{kg} / \mathrm{m}^{3}$ \\
\hline
\end{tabular}

Fonte: Autores

Para auxiliar na simulação pelo "Método Analítico" e "Método Numérico", duas calculadoras estão disponíveis no canto inferior direito da interface do simulador para as estimativas iniciais dos parâmetros $\beta$ e $\gamma$. Como o valor de $\beta$ depende apenas do meio (ar) e das dimensões do objeto, normalmente, esse valor é inserido na calculadora central e não exige ajustes posteriores. Para a estimativa inicial de $\gamma$, recomenda-se inserir $C=0,5$ no caso de um objeto esférico. A ideia central da simulação é inserir, por tentativas, valores de $\beta$ e $\gamma$ que resulte em uma curva simulada que seja compatível com a curva experimental. Portanto, uma vez que as curvas simuladas e experimentais estejam alinhadas, é preciso calcular o valor experimental de $C$ a partir da Equação (12), utilizando o valor de $\gamma$ simulado.

Após a finalização de uma análise, é possível salvar os dados de uma curva simulada inserindo no campo do "NOME" a simulação que se deseja exportar e clicando no botão "EXPORTAR" - um arquivo com o nome da simulação no formato .csv é criado na pasta do simulador.

\section{RESULTADOS E DISCUSSÃO}

O programa desenvolvido neste trabalho permite simular uma curva teórica em cima de dados experimentais, possibilitando a determinação do coeficiente de arrasto do objeto lançado, bem como o efeito da resistência do ar no movimento. Por essa razão, é muito 
fácil visualizar os casos em que a resistência do ar pode ser considerada desprezível e casos em que ela desempenha um papel importante na dinâmica do movimento de uma bola. Essa simulação também permitiu estabelecer que nem todos os modelos matemáticos considerando a resistência do ar são adequados para a descrição do movimento.

A Figura 2 mostra as curvas experimental e simuladas sem resistência do ar e com resistência do ar pelo "Método Analítico" e pelo "Método Numérico" para a bola de plástico. Podemos observar uma boa adequação do modelo que considera $F_{v} \propto v, v^{2}$, pelo "Método Numérico". Entretanto, o modelo com $F_{v} \propto v$, com o "Método Analítico", não se adequa aos dados experimentais, que parece sobrepor com a simulação sem resistência do ar pela ampliação mostrada. Isso ocorre também para as bolas de isopor e vôlei em que a resistência do ar afeta significativamente o movimento. Esse resultado está de acordo com os trabalhos de Lock (1982) e Aguiar e Rubini (2004), devido à faixa de valores de velocidade alcançada pelas bolas no presente experimento.

Figura 2 - Simulação para a bola de plástico.
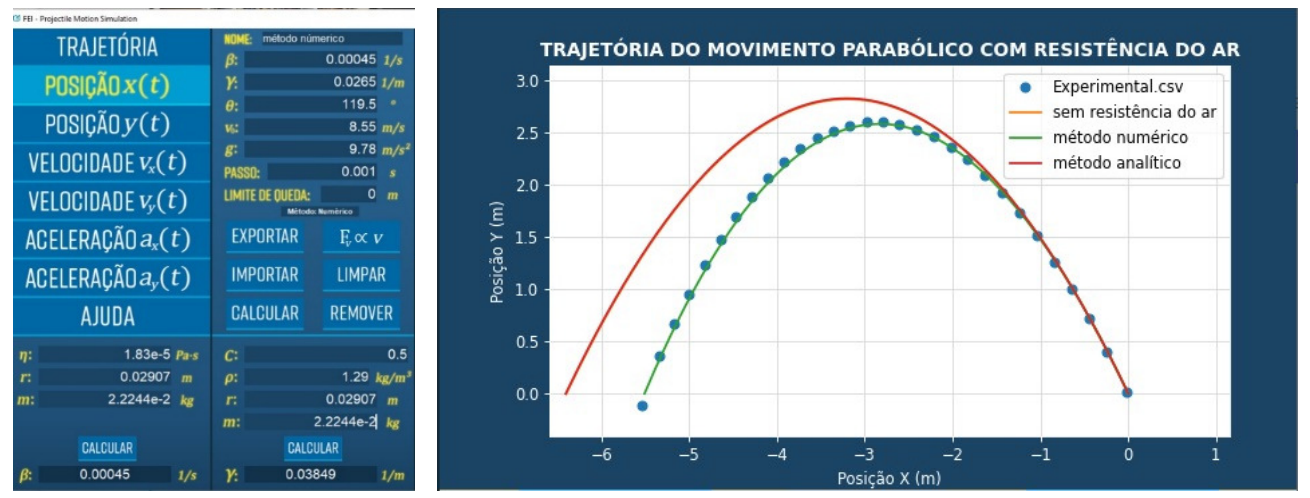

Fonte: Autores

Os resultados das análises dos movimentos para as cinco bolas estudadas são mostrados nas Figuras (3) - (7). São os resultados das simulações desprezando e considerando a resistência do ar pelo "Método Numérico" para as curvas da trajetória, de posição $[x(t)$ e $y(t)]$ e velocidade $\left[v_{x}(t)\right.$ e $\left.v_{y}(t)\right]$. Os dados de aceleração não são mostrados por não permitirem uma avaliação adequada, devido à significativa flutuação. $O$ estudo da trajetória das cinco bolas diferentes através da simulação mostra que existirão situações em que a resistência do ar pode ou não ser desprezada. Para as condições experimentais deste estudo, em que o alcance, a altura e a velocidade inicial não ultrapassaram $6 \mathrm{~m}, 3 \mathrm{~m}$ e $9 \mathrm{~m} / \mathrm{s}$, respectivamente, podemos considerar que a resistência do ar é desprezível para os movimentos das bolas de futebol e basquete, em que as curvas experimentais e simuladas sem resistência do ar estão bem justapostas. Por outro lado, os resultados para as bolas de isopor, plástico e vôlei indicam claramente que a resistência do ar deve ser considerada para a descrição do movimento. Observamos uma boa concordância entre as curvas simuladas considerando a resistência do ar e as experimentais dessas três bolas. A partir dos valores de $\gamma$ utilizadas na simulação mostradas nas Figuras (3) - (5), calculamos o coeficiente de arrasto $C$ utilizando a Equação (12), que resultou nos valores $0,41,0,34$ e 0,35 para as bolas de isopor, plástico e vôlei, respectivamente.

Figura 3 - Curvas experimentais (pontos) e simuladas (linhas) para a bola de isopor. 

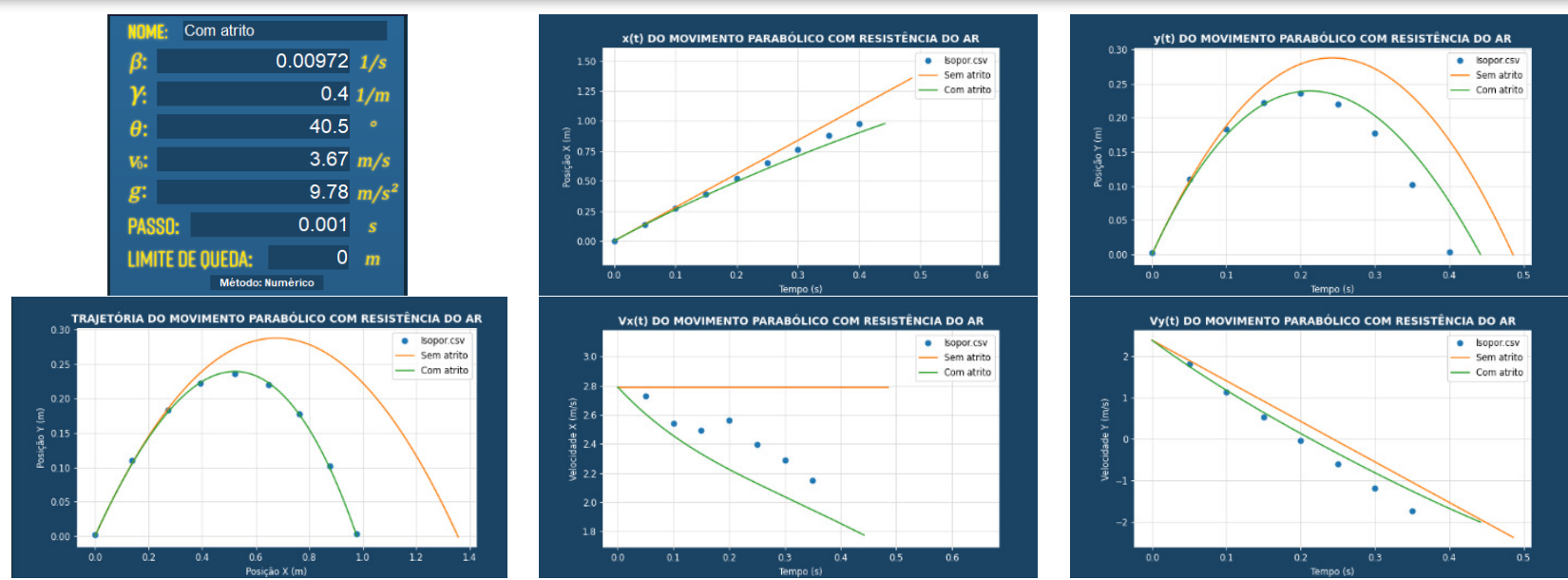

Fonte: Autores

Figura 4 - Curvas experimentais (pontos) e simuladas (linhas) para a bola de plástico.
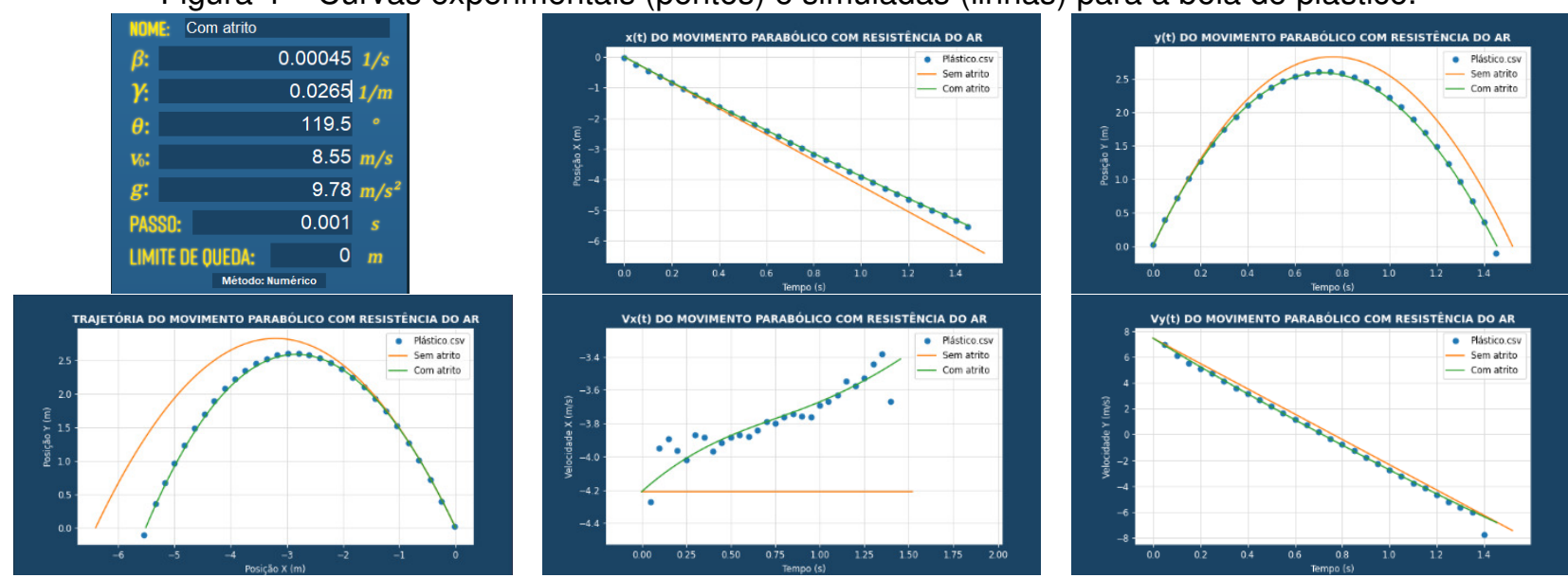

Fonte: Autores

A comparação entre dados experimentais e simulados com diversos modelos matemáticos ajuda na percepção sobre a relevância (ou não) de certos parâmetros físicos de um problema real. Sem isso, algumas vezes, essa percepção não é trivial. Por exemplo, ao analisarmos as trajetórias das bolas de futebol e basquete, concluímos que a resistência do ar poderia ser considerada desprezível nas equações de movimento. Assim, poderíamos esperar o mesmo para a bola de vôlei, com diâmetro menor, contudo, o resultado da análise mostra que o efeito da resistência do ar foi relevante para descrever a trajetória da bola de vôlei.

Os resultados da simulação para as bolas de isopor e vôlei mostrados nas Figuras (3) e (5), ainda indicam que outros fatores, além da resistência do ar, devem ser considerados quando as situações experimentais não são ideais. Podemos observar que as curvas simuladas e experimentais não são completamente compatíveis. Por exemplo, a rotação das bolas, difícil de ser evitada num lançamento com as mãos, não foi considerada na equação de movimento. É possível que as diferenças observadas também sejam resultantes de condições do ambiente, como correntes de ar, que são difíceis de controlar. Erros experimentais também podem ocorrer durante a filmagem da trajetória, que deve ser feita no plano do movimento, pois o Tracker analisa o movimento apenas em duas dimensões. Entretanto, minimizar esses erros pode não ser uma tarefa fácil para um lançamento de alguns metros. 
Figura 5 - Curvas experimentais (pontos) e simuladas (linhas) para a bola de vôlei.
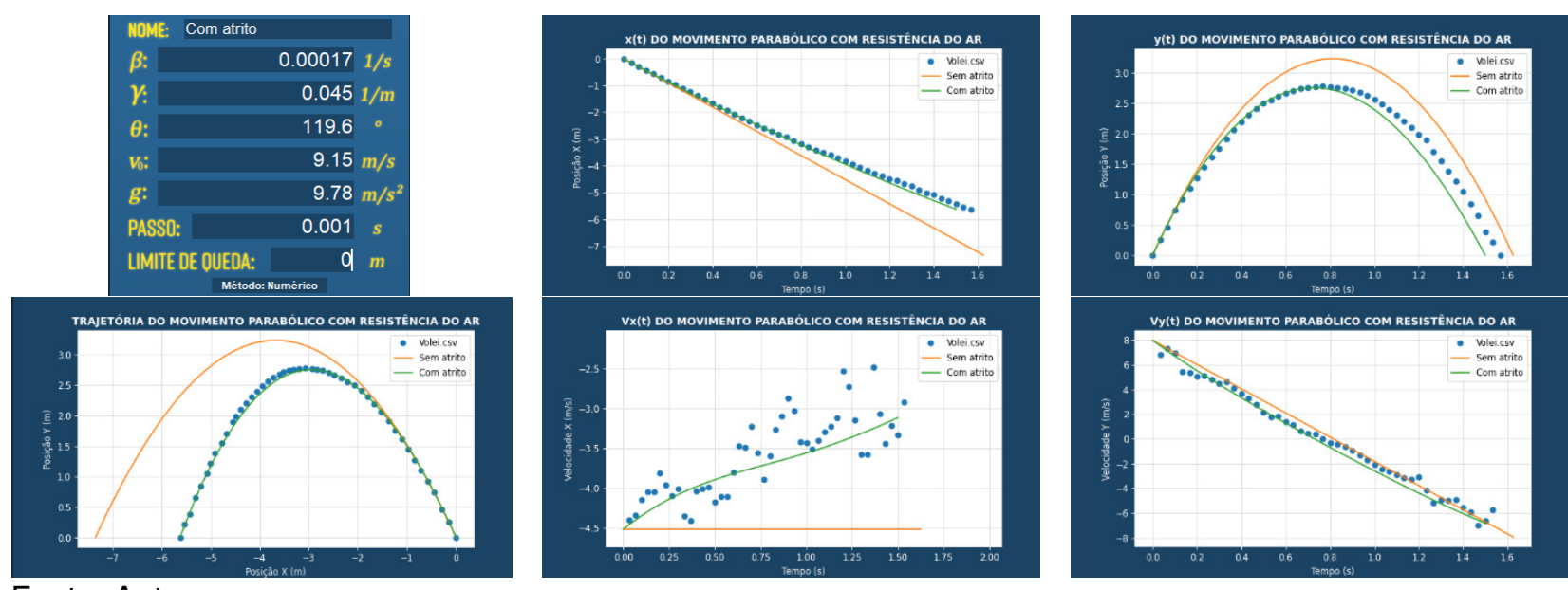

Fonte: Autores

Figura 6 - Curvas experimentais (pontos) e simuladas (linhas) para a bola de futebol.
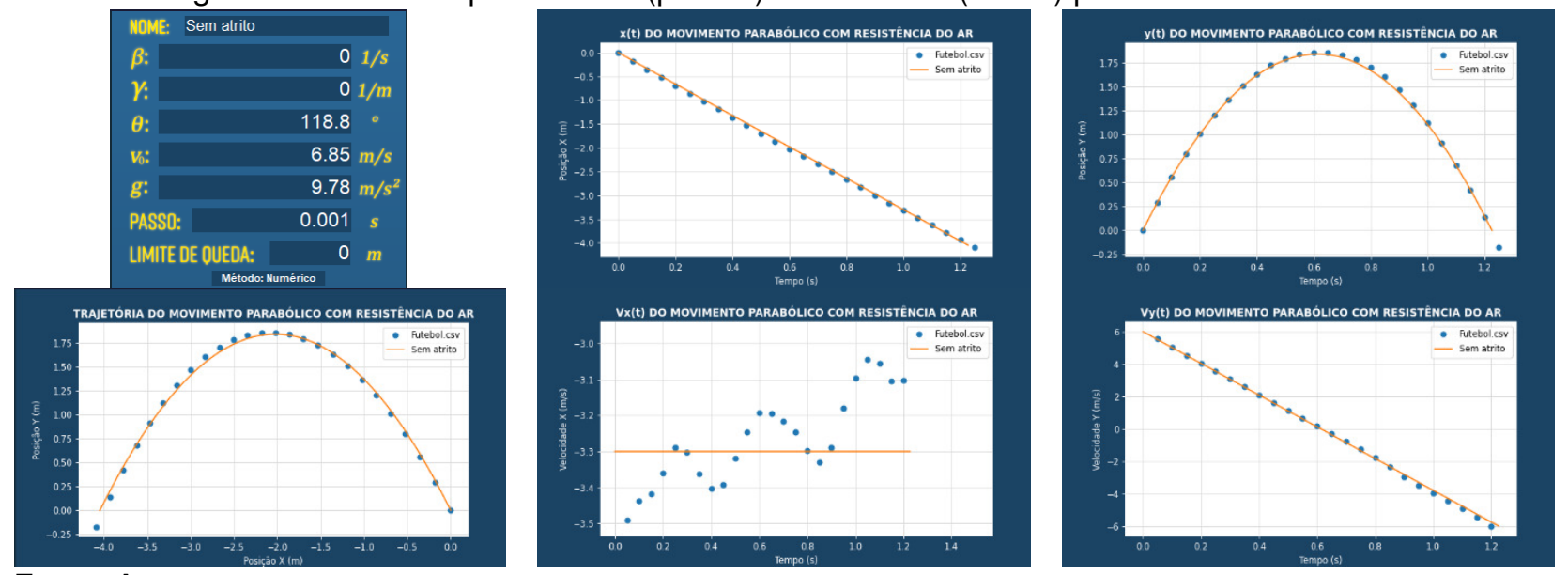

Fonte: Autores

Os resultados levemente divergentes entre simulações e experimentos também podem contribuir para uma reflexão crítica por parte dos estudantes, que os levam a questionar se é apenas uma questão de repetir o experimento em condições mais controladas ou pesquisar outros efeitos que não foram inicialmente considerados na simulação, como a rotação da bola e a crise do arrasto, que é observado para bolas com velocidades maiores, tipicamente alcançadas por bolas lançadas por profissionais do esporte (AGUIAR; RUBINI, 2004). Essa análise crítica pode contribuir para o próprio aprendizado do aluno e para o desenvolvimento das competências associadas à aplicação de métodos científicos para a análise de fenômenos físicos; à condução de experimentos e interpretação de resultados; e à modelagem matemática de sistemas físicos. Em função das análises realizadas, os alunos podem avaliar os limites de aplicabilidade de modelos teóricos e da própria simulação, possibilitando a reflexão crítica do procedimento e análise experimental ao longo da experiência.

Figura 7 - Curvas experimentais (pontos) e simuladas (linhas) para a bola de basquete. 

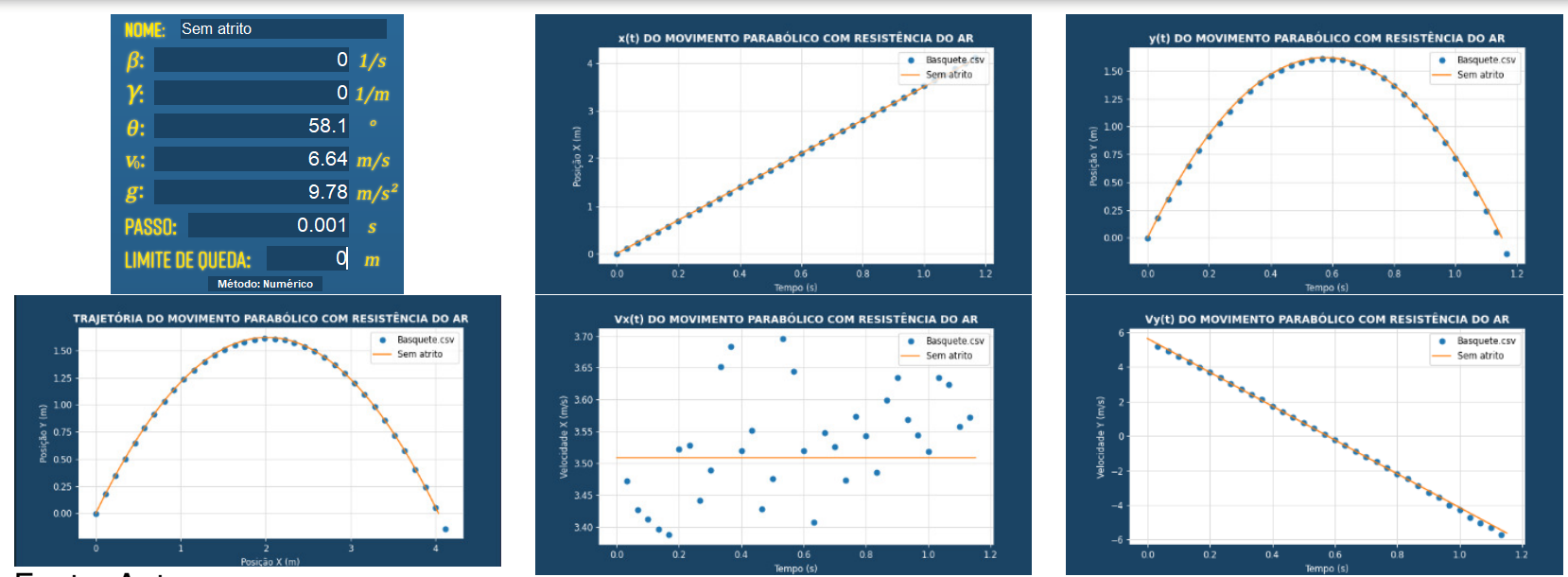

Fonte: Autores

Ainda que a ênfase tenha sido dada à grande vantagem da possibilidade de comparação entre dados experimentais e simulados, o professor pode optar por: ( $I$ ) analisar o programa em si (opção mais computacional); (ii) analisar com mais detalhes as equações utilizadas no método analítico onde a $F_{v} \propto v$ e sua aplicabilidade; e/ou (iii) abordar o método numérico para solução de equações diferenciais não lineares e sua aplicabilidade, promovendo a interdisciplinaridade.

\section{CONSIDERAÇÕES FINAIS}

Com o uso da simulação, foi possível fazer um estudo eficiente e bem aprofundado sobre o movimento balístico - comumente, a resistência do ar não é abordada em sala de aula. Essa barreira pode ser contornada utilizando a simulação e o aluno pode desenvolver competências que o prepararão para resolver problemas de maneira mais autônoma e se responsabilizando pelo seu aprendizado. Além de auxiliar no desenvolvimento de competências, a participação do aluno em atividades experimentais e colaborativas facilita o desenvolvimento da aprendizagem significativa, aproveitando os conhecimentos prévios do aluno (MOREIRA, 2019), uma vez que o lançamento de projétil é listado como um dos componentes curriculares do Ensino Médio. Para isso, também é preciso atentar para que o aluno compreenda cada um dos parâmetros introduzidos para o cálculo e reflita sobre os resultados da simulação. Desta forma, o aluno terá maior compreensão do problema físico e dos fatores relevantes para a descrição do movimento, refletindo sobre os conceitos e os fenômenos abordados.

Apresentamos uma proposta de atividade em que aliamos a experimentação, o modelo matemático e a simulação visando o desenvolvimento de competências contempladas nas Diretrizes Curriculares Nacionais do Curso de Graduação em Engenharia, desde o início do curso de engenharia, por alunos ingressantes. Com o advento da TIC, é possível desenhar atividades para o ensino de física com baixo custo e adaptáveis ao ambiente doméstico, mas com alta qualidade acadêmica. Esperamos que essa atividade também contribua para um aprendizado significativo do estudante, uma vez que são considerados o seu conhecimento prévio e envolve uma discussão que possui um certo apelo didático por considerar a resistência do ar nas equações de movimento balístico e consequente abordagem de diferentes modelos matemáticos para esse fim.

\section{Agradecimentos}


Este trabalho teve apoio financeiro da Fundação Educacional Inaciana Pe. Sabóia de Medeiros (Bolsa de iniciação didática PRO-BID029/19). Os autores agradecem ao Prof. Dr. Roberto Baginski pelas sugestões ao longo do desenvolvimento do trabalho.

\section{REFERÊNCIAS}

AGUIAR, Carlos Eduardo; RUBINI, Gustavo. A aerodinâmica da bola de futebol. Revista Brasileira de Ensino de Física, v. 26, n. 4, p. 297-306, 2004.

ANACONDA. Anaconda Navigator - Anaconda Documentation. [S.I.]: Anaconda, Inc. Disponível em: https://docs.anaconda.com/anaconda/navigator/. Acesso em: 21 mar. 2021.

CNE, Conselho Nacional de Educação. Câmara de Educação Superior. Diretrizes Curriculares Nacionais do Curso de Graduação em Engenharia. Ministério da Educação. Brasil. Resolução CNE/CES n 2, de 24 de Abril de 2019.

DORNELES, Pedro FT; ARAUJO, Ives S.; VEIT, Eliane A. Simulação e modelagem computacionais no auxílio à aprendizagem significativa de conceitos básicos de eletricidade: parte l-circuitos elétricos simples. Revista Brasileira de Ensino de Física, v. 28, n. 4, p. 487-496, 2006.

FERREIRA, G. F. Projéteis com Resistência do Ar Quadrática na Velocidade. Revista Brasileira de Ensino de Física, v. 23, n. 3, p. 271-275, 2001.

FREIRE, Wilson Hugo C. et al. Lançamento oblíquo com resistência do ar: Uma análise qualitativa. Revista Brasileira de Ensino de Física, v. 38, n. 1, p. 1306.1-1306.5, 2016.

HALLIDAY, David; RESNICK, Robert; WALKER, Jearl. Fundamentos de Física, Volume 1: Mecânica. 10. ed. Rio de Janeiro: LTC - Livros Técnicos e Científicos Editora Ltda, 2016.

HALVERSON, E.; SHERIDAN, K. The maker movement in education. Harvard Educational Review, v. 84, n. 4, p. 495-504, 2014.

HOFFMAN, Joe D.; FRANKEL, Steven. Numerical methods for engineers and scientists. 2. ed. Boca Raton: CRC press, 2018.

LOCK, James A. The physics of air resistance. The Physics Teacher, v. 20, n. 3, p. 158160, 1982.

MOREIRA, M. A. O que é afinal Aprendizagem Significativa? Qurriculum, La Laguna, n. 25, 2012.

PALIERINI, R. Z. FEI-Projectile-Motion-Simulation. Disponível em:

https://github.com/Zakonildo/FEl-Projectile-Motion-Simulation. Acesso em: 20 abr. 2021

PHYSICS, Open Source. Tracker - Video Analysis and Modeling Tool. [S.I.]: Open 
Source Physics. Disponível em: https://physlets.org/tracker/. Acesso em: 21 mar. 2021.

PYTHON. Python 3.8.5 Documentation. [S.I.: s.n.]. Disponível em:

https://docs.python.org/3/. Acesso em: 21 mar. 2021.

ROGERS, Carl Ransom. Um jeito de ser. São Paulo: EPU, 1983.

SRINATH, K. R. Python-the fastest growing programming language. International

Research Journal of Engineering and Technology, v. 4, n. 12, p. 354-357, 2017.

\title{
PYTHON-BASED SIMULATION AND VIDEO ANALYSIS OF BALLISTIC MOTION WITH AIR RESISTANCE
}

\begin{abstract}
The aim of this work is to present an activity for freshman students that is in line with the development of skills in engineering training. We propose a study on the effect of air resistance on the ballistic motion of some objects through video analysis and Pythonbased simulations to determine if drag force is relevant or not in each case. In order to illustrate the procedure, we have evaluated the results for five different balls launched obliquely. The combined visualization of the experimental data and the trajectory simulation allowed us to establish the influence of air resistance in the balls trajectory. Air resistance was negligible on the movement of the basketball and the football. However, we observe that the motion of the styrofoam, plastic and volley balls was under the influence of air resistance forces. In these cases, it was possible to estimate the drag coefficient of the styrofoam, plastic and volley balls by using the simulation.
\end{abstract}

Keywords: Python-based simulation. Ballistic motion. Drag force. 